\title{
Grazing and Hopf Bifurcations of a Periodic Forced System with Soft Impacts
}

\author{
Xifeng Zhu ${ }^{*}, 1$ and Quanfu $\mathrm{Gao}^{2}$

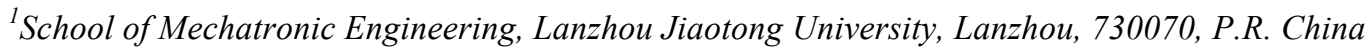 \\ ${ }^{2}$ Key Laboratory of System Dynamics and Reliability of Rail Transport Equipment of Gansu Province, Lanzhou, 730070, \\ P.R. China
}

\begin{abstract}
Based on the research of a periodic forced system with soft impacts, the piecewise properties of the softimpacts system, such as asymmetric motion and singularity, were analyzed by using the Poincaré map and Runge-Kutta numerical simulation method. The routes from periodic motions to chaos, via Hopf bifurcation and grazing bifurcation, were investigated thoroughly. In the case of large constraint stiffness, the Hopf bifurcation is observed in the periodic forced system with soft impacts. The clearances of the system are the main reasons for influencing the chaotic motion. For small clearances, the grazing bifurcations bring about asymmetric motion and singularity. The steady 1-1-1 period orbits will exist within a wideband frequency range when appropriate system parameters are chosen.
\end{abstract}

Keywords: Grazing bifurcation, soft impacts, periodic motion, vibration

\section{INTRODUCTION}

The soft impacts induce extensive oscillation in mechanical systems. Such piecewise linear systems are capable of exhibiting classically non-linear behavior such as grazing bifucations. For example, impact dampers, shakers, etc., are based on the impact of moving bodies. With other s, mechanisms with clearances, gears, wheel-rail interaction of railway coaches, etc., impacts also occur, but they are undesirable as they bring about increased wear and noise levels. It is necessary to be able to accurately model the dynamics of mechanical systems with soft impacts and clearances, to enlarge profitable effects and minimize adverse effects. The grazing bifurcation, which is a key to determine the motions switching in discontinuous dynamical systems, has been discussed by a number of researches in the past several years. Shaw and Holmes [1] studied a singledegree-of-freedom vibro-impact system by using the traditional approaches for analyzing periodic-impact responses in the system. The results showed all types of typical nonlinear behavior: saddle node and flip bifurcations, multiple coexisting attracting solutions and chaos, etc. Nordmark [2] developed systematic methods for investigating grazing dynamics and attendant bifurcations of the piecewise linear and vibro-impact systems. A study De Souza and Wiercigroch [3] focused on the grazing transitions from no impact to impact motion and investigated parameter space region around the grazing bifurcations. The qualitative changes that can be associated with grazing and corner-collision bifurcations were observed through a combination of experiments and numerical calculations

*Address correspondence to this author at the School of Mechatronic Engineering, Lanzhou Jiaotong University, Box no.406, 88 West Anning Road, Lanzhou, Gansu Province, Postcard: 730070, P.R. China;

Tel: 0086-931-4938143; E-mail: zhuxf@mail.lzjtu.cn
Long et al., Luo and Gegg [4, 5] carried out a comprehensive investigation on grazing motions in the dry-friction oscillator for a better understanding of the grazing mechanism of a discontinuous system. Experimental study on piecewise linear oscillator was performed by Sin and Wiercigroch [6]. Luo and O'Connor [7, 8] presented an idealized, piecewise linear system to model non-smooth vibration of gear transmission appearing from impacts between the gear teeth. For example, in wheel-rail impacts of railway coaches Luo et al. [9], Jeffcott rotor with bearing clearance Karpenko et al. [10], gears transmissions A1shyyab, and Kahraman [11], small vibro-impact pile driver Luo and Yao [12], etc., impacting models have been proved to be useful.

A periodic forced system with soft impacts and clearances has been established. The main purpose of the present study was to analyze the piecewise properties of such system, including stability, Hopf bifurcation, grazing bifurcations period doubling bifurcation, etc. The routes from quasi-periodic impact motions, grazing motions or period-doubling cascades to chaos were observed by using the Poincaré map and numerical simulation. Finally, the influences of clearances on periodic motions and bifurcations of the periodic forced system are discussed in detail.

\section{MECHANICAL MODEL}

The mechanical model of a periodic forced system with soft impacts is shown in Fig. (1). Displacements of the masses $M_{1}, M_{2}$ and $M_{3}$ are described by $X_{1}, X_{2}$ and $X_{3}$, respectively. The mass $M_{1}$ and $M_{2}$ are connected by linear spring with stiffness $K_{1}$ and linear viscous dashpot with damping constant $C_{1}$, and $M_{2}$ and $M_{3}$ are connected by $K_{2}$ and $C_{2}$ analogously. The mass $M_{3}$ is attached to the supporting base by the linear spring with stiffness $K_{3}$ and 
linear viscous dashpot with damping constant $C_{3}$. The excitations on masses are harmonic with amplitudes $P_{1}, P_{2}$ and $P_{3} . \Omega$ is the excitation frequency, and $\tau$ is the phase angle. The mass $M_{2}$ begins to hit the right (left) soft impact represented by linear spring with stiffness $K_{4}\left(K_{5}\right)$ and linear viscous dashpot with damping constant $C_{4}\left(C_{5}\right)$ when the displacement $X_{2}$ of mass $M_{2}$ equals the clearances $B$, i.e. $X_{2}(t)=B\left(X_{2}(t)=-B\right)$.

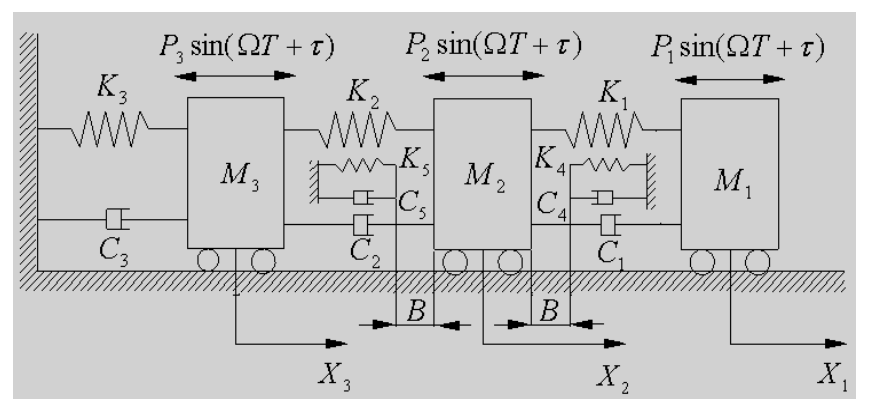

Fig. (1). Mechanical model of a periodic forced system with soft impacts.

The motion processes of the system when the absolute value of displacement $X_{2}$ is less than the clearances are considered. The condition of the periodic forced system, just immediately after impact, is observed as initial condition in the subsequent process of the motion. The non-dimensional differential equations of motion are given by Eq.(1) (4).

$$
\begin{gathered}
\ddot{x}_{1}+2 \xi\left(\dot{x}_{1}-\dot{x}_{2}\right)+\left(x_{1}-x_{2}\right)=f_{10} \sin (\omega t+\tau) \\
\mu_{m 2} \ddot{x}_{2}+2 \xi\left(1+\mu_{c 2}\right) \dot{x}_{2}-2 \xi \dot{x}_{1}-2 \xi \mu_{c 2} \dot{x}_{3}-\mu_{k 2} x_{3} \\
+\left(1+\mu_{k 2}\right) x_{2}-x_{1}+f\left(x_{2}\right)=f_{20} \sin (\omega t+\tau) \\
\mu_{m 3} \ddot{x}_{3}+2 \xi\left(\mu_{c 2}+\mu_{c 3}\right) \dot{x}_{3}-2 \xi \mu_{c 2} \dot{x}_{2}+\left(\mu_{k 2}+\mu_{k 3}\right) x_{3} \\
-\mu_{k 2} x_{2}=\left(1.0-f_{10}-f_{20}\right) \sin (\omega t+\tau) \\
f\left(x_{2}\right)= \begin{cases}2 \xi \mu_{c 4} \dot{x}_{2}+\mu_{k 4}\left(x_{2}-b\right), & x_{1}>b, \\
0 & -b \leq x_{1} \leq b, \\
2 \xi \mu_{c 5} \dot{x}_{2}+\mu_{k 5}\left(x_{2}+b\right), & x_{1}<-b .\end{cases}
\end{gathered}
$$

where the non-dimensional quantities are given by

$$
\begin{aligned}
& \mu_{m i}=\frac{M_{i}}{M_{1}}, \mu_{k j}=\frac{K_{j}}{K_{1}}, \mu_{c j}=\frac{C_{j}}{C_{1}}, f_{i 0}=\frac{P_{i}}{P_{1}+P_{2}+P_{3}}, \\
& b=\frac{B K_{1}}{P_{1}+P_{2}+P_{3}}, x_{i}=\frac{X_{i} K_{1}}{P_{1}+P_{2}+P_{3}}, \omega=\Omega \sqrt{\frac{M_{1}}{K_{1}}} \\
& t=T \sqrt{\frac{K_{1}}{M_{1}}}, \xi=\frac{C_{1}}{2 \sqrt{K_{1} M_{1}}} i=2,3,4,5, j=1,2,3
\end{aligned}
$$

Periodic-impact motions of the system are described by the symbol $n-p-q$, where $n$ denotes the number of excitation periods and $p(q)$ denotes the number of impacts with right (left) soft impacts, during one impact motion period, respectively. In order to establish the Poincaré map of the periodic forced system, we chose the Poincaré section: $\sigma=\left\{\left(x_{1}, \dot{x}_{1}, x_{2}, \dot{x}_{2}, x_{3}, \dot{x}_{3}, t\right) \in R^{6} \times S, x_{2}=b, \dot{x}_{2}>0\right\}$. The disturbed map of period $n$ single-impact motion is expressed briefly by

$\mathbf{X}^{\prime}=\tilde{f}(v, \mathbf{X})$

where $X \in \mathrm{R}^{6}, v$ is real parameters, $v \in \boldsymbol{R}^{m} ; X=X^{*}+\Delta X$, $X^{\prime}=X^{*}+\Delta X^{\prime}, X^{*}=\left(x_{10}, \dot{x}_{1 p}, \dot{x}_{2 p}, x_{30}, \dot{x}_{3 p}, \tau_{0}\right) \quad \mathrm{T}$ is a fixed point in the hyperplane $\sigma$, $\Delta X=\left(\Delta x_{10}, \Delta \dot{x}_{1 p}, \Delta \dot{x}_{2 p}, \Delta x_{30}, \Delta \dot{x}_{3 p}, \Delta \tau\right)^{\mathbf{T}} \quad$ and $\quad \Delta X^{\prime}=$ $\left(\Delta x_{10}^{\prime}, \Delta \dot{x}_{1 p}^{\prime}, \Delta \dot{x}_{2 p}^{\prime}, \Delta^{\prime} x_{30}, \Delta \dot{x}_{3 p}^{\prime}, \Delta \tau^{\prime}\right)^{\mathrm{T}}$ are the disturbed vectors of $X^{*}$.

\section{GRAZING BIFURCATIONS AND PERIODIC MOTIONS}

The existence and stability of $n-p-q$ motions have been analyzed explicitly. Bifurcations at the points of change in stability are considered, thus giving some information about the existence of different kinds of bifurcations named periodic-doubling bifurcation, Hopf bifurcation, grazing bifurcation, etc.

(a) global bifurcation

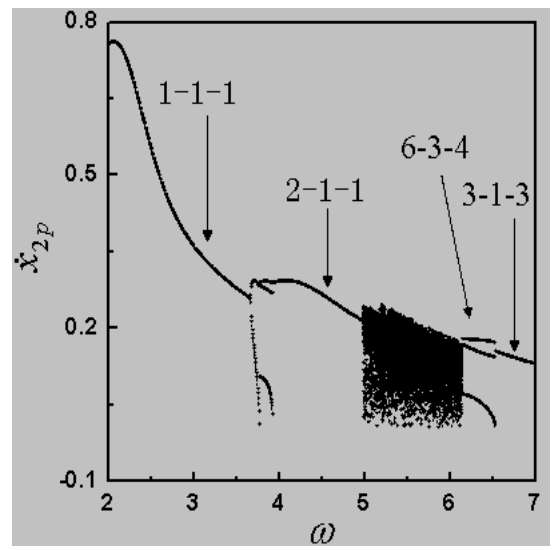

(b) detail of Fig. (2a)

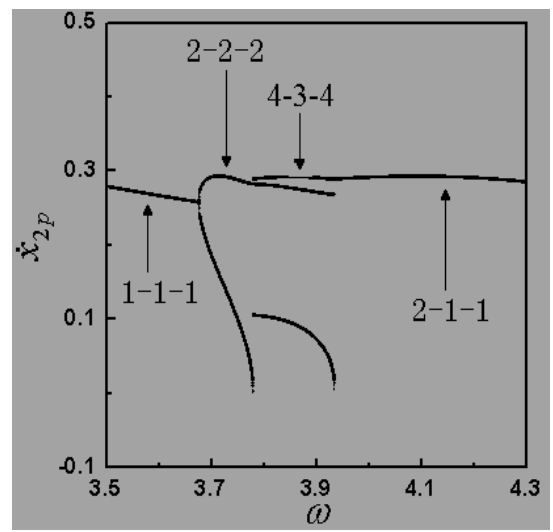

Fig. (2). Bifurcation diagrams.

Taking dimensionless parameters (1): $b=0.01, \mu_{k 2}=2.0$, $\mu_{k 3}=5.0, \mu_{k 4}=50.0, \mu_{k 5}=0.1, \xi=0.2, \mu_{c 2}=2.0, \mu_{c 3}=2.0$, $\mu_{c 4}=2.0, \mu_{c 5}=1.0, \mu_{m 2}=1.0, \mu_{m 2}=1.0, f_{10}=0.0, f_{20}=$ 
1.0 , to analyze the nonlinear dynamic performance of the soft-impact system. The global bifurcations of the softimpact system, in the form of projected Poincaré section, is shown for $\omega$ varying in the range [2.0, 7.0], see Fig. (2a). Fig. (2b) shows detail of the local bifurcations of Fig. (2a). It is shown that the system exhibited stable 1-1-1 motion with $\omega \in[2.0,3.6912)$. As $\omega$ passed through $\omega_{c}=3.6912$ increasingly, the 1-1-1 motion changed its stability. Moreover, period-doubling bifurcation was associated with the motion so that the 2-2-2 impact motion could be generated. Stable 2-2-2 motion existed in the frequency interval $\omega \in(3.6912,3.778)$; a phase portrait of 2-2-2 motion is shown for $\omega=3.772$ in Fig. (3a, b). When $\omega$ increased to $\omega=3.778$, the mass $M_{2}$ began to touch the right soft impacts with zero velocity and the grazing bifurcation took place, while the stable 4-3-4 motion was observed immediately after the grazing bifurcation. Figs. (3c, d) showed the 2-2-2 motion with grazing contact for $\omega=3.778$. Stable 4-3-4 motion was observed in the frequency interval $\omega \in(3.778,3.9246)$, with a phase portrait of 4-3-4 motion shown for $\omega=3.84$ in Fig. (4a). With increase in the forcing frequency, the system exhibited the 2-1-1 motion for $\omega \in(3.9246,4.9145)$ and the 2-1-1 motion is shown for $\omega=4.2$ in Fig. (4b). After chaos, there was 6-34 motion observed at $\omega=6.2$, and Figs. (4c, d) showed the 3-1-3 motion at $\omega=6.5$.

\section{HOPF BIFURCATION AND QUASI-PERIODIC ATTRACTOR}

The system parameters (2): $b=0.1, \mu_{k 2}=1.0, \mu_{k 3}=1.0$, $\mu_{k 4}=50.0, \mu_{k 5}=50.0, \xi=0.1, \mu_{c 2}=0.2, \mu_{c 3}=2.0, \mu_{c 4}=$ 2.0, $\mu_{c 5}=0.0, \mu_{m 2}=1.0, \mu_{m 2}=2.0, f_{10}=0.0$, and $f_{20}=$ 1.0, were chosen for the analysis. In Fig. (5a) the results from simulation, in the form of projected Poincaré section, are shown for $\omega$ varying in the range [2.7, 2.9].

It is shown that the system exhibited stable 1-1-1 motion with $\omega \in(2.8351,2.9)$. As $\omega$ passed through $\omega_{c}=2.8351$ decreasingly, the 1-1-1 motion changed its stability, and Hopf bifurcation associated with the motion occurred so that the quasi-periodic impact motions could take place. As expected, the 1-1-1 motion is represented by one fixed point in projected portrait of Poincare' map, appeared at $\omega=$ 2.8351, as shown in Fig. (5b). Quasi-periodic response, represented by the attracting invariant circle is shown in Fig. (5c), which appeared at $\omega=2.82$, just after the Hopf bifurcation. With decrease in the forcing frequency, the system exhibited the 3-3-3 motion, and Fig. (5d) shows the attracting invariant circle at $\omega=2.774$ and $3-3-3$ motion at $\omega=2.75$.

\section{THE INFLUENCE OF CLEARANCES ON PERIODIC MOTIONS AND BIFURCATIONS}

The influence of clearances between the system parts on dynamics is presented in the study. Taking system parameters (1): (a) 2-2-2 motion, $\omega=3.772$

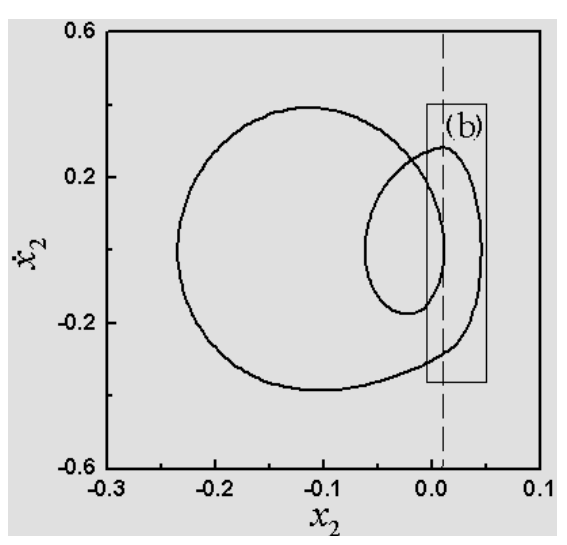

(b) detail of Fig. (3a)

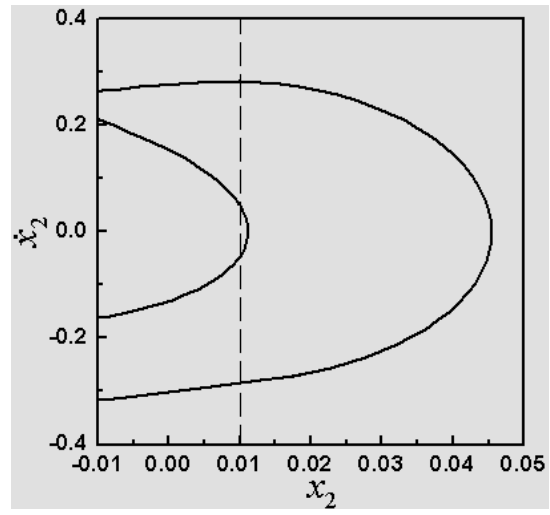

(c) 2-2-2 motion with grazing contact, $\omega=3.778$

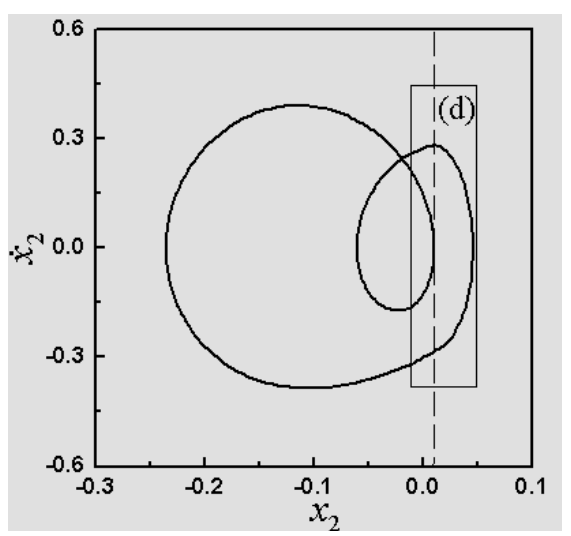

(d) detail of Fig. (3c)

Fig. (3). Phase portraits. 
(a) 4-3-4 motion, $\omega=3.84$

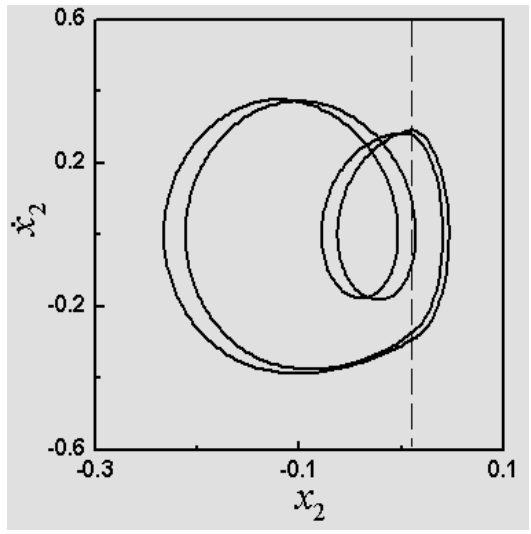

(b) 2-1-1 motion, $\omega=4.2$

(c) 6-3-4 motion, $\omega=6.2$

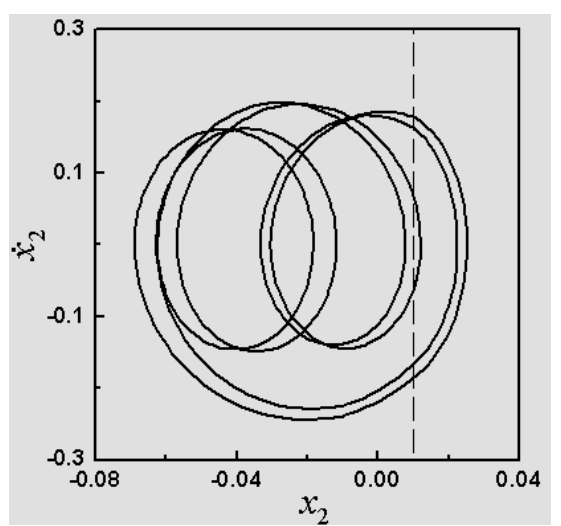

(d) 3-1-3 motion, $\omega=6.5$

Fig. (4). Phase portraits (a) bifurcation diagram

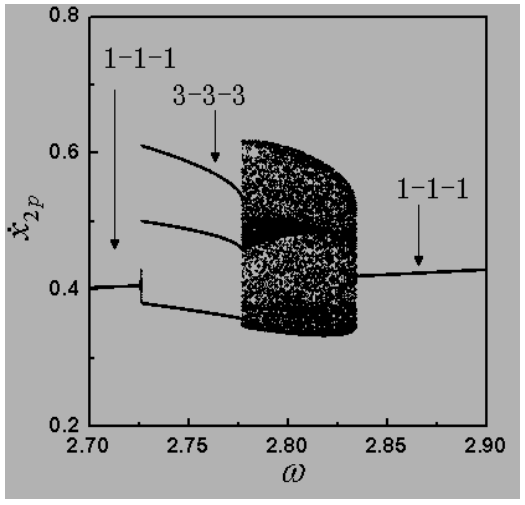

(b) fixed point, $\omega=2.8351$

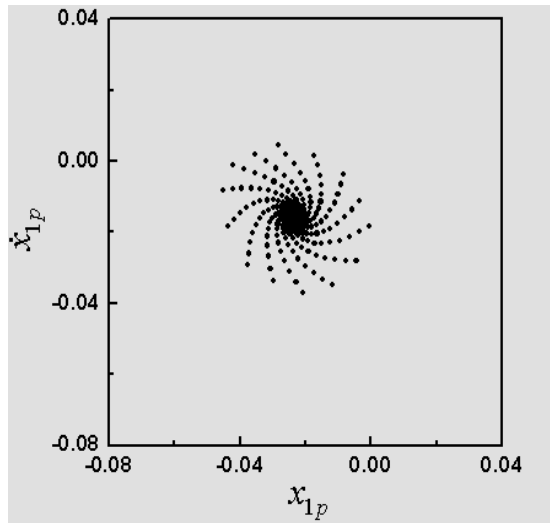

(c) quasi-periodic motion, $\omega=2.82$

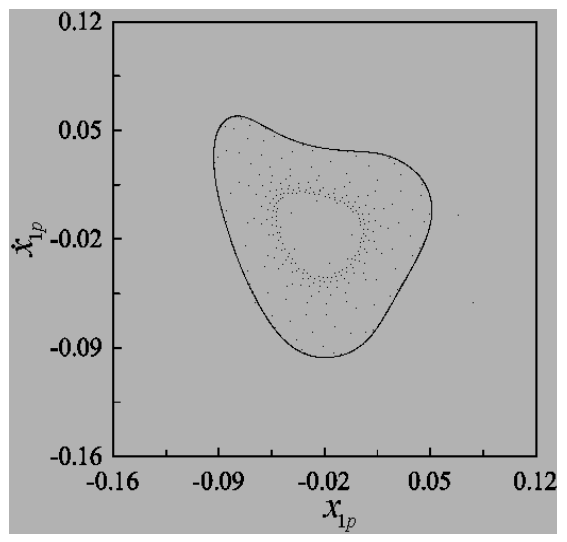

(d) quasi-periodic motion, $\omega=2.774$ and 3-3-3 motion, $\omega=2.75$

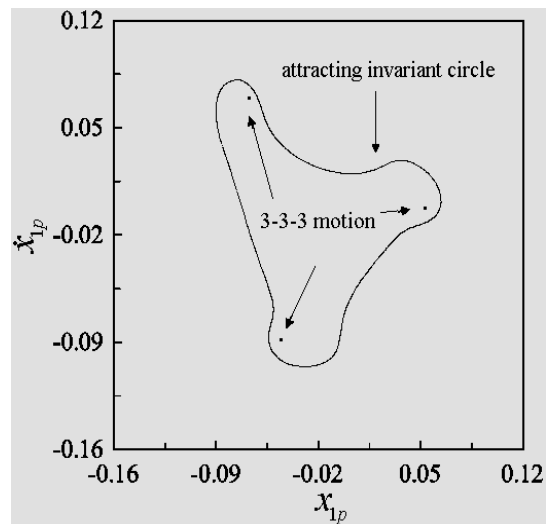

Fig. (5). Bifurcation diagram and projected Poincaré maps. 
$b=0.01, \mu_{k 2}=2.0, \mu_{k 3}=5.0, \mu_{k 4}=50.0, \mu_{k 5}=0.1, \xi=$ $0.2, \mu_{c 2}=2.0, \mu_{c 3}=2.0, \mu_{c 4}=2.0, \mu_{c 5}=1.0, \mu_{m 2}=1.0$, $\mu_{m 2}=1.0, f_{10}=0.0$, and $f_{20}=1.0$, as the criterion parameters, to analyze the influence of clearances between the system parts on periodic motions and bifurcations. Fig. (6) shows the bifurcation diagrams for the impact velocity $\dot{x}_{2 p}$ of the periodic forced system under the conditions of different clearance parameters. Only changed parameter is given in Fig. (6a-d), and all the other parameters, not listed in the figure's description, were the same as the criterion parameters.

The effects of changes in clearances $b$ were analyzed by changing their value. Large or small clearances slightly influenced the velocity amplitude of the periodic motion, which can be seen in Fig. (6a-d). The minimum value of clearances, i.e. $b=0.0$, led to small change in the topological structure of the bifurcations, as shown in Fig. (6a). As the value of $b$ increased, the frequency range of 1-1-1 motion became narrow and period-doubling bifurcations sequence was observed, such as 1-1-1 motion and 2-2-2 motion for large excitation frequency as shown in Fig. (6b). Large value of $b$ resulted in the narrow frequency ranges of 1-1-1 motion, moving toward small $\omega$. The singularities and asymmetric periodic motions such as 2-1-2, 3-2-3, and 4-3-4 motions are indicated in Fig. (6c). A slightly larger value of clearances was considered as shown in Fig. (6d), and the dynamic properties of the periodic forced system were simple. As shown in Fig. (6c, d), for large clearances, the mass $M_{2}$ did not contact the soft impacts at high excitation frequency.

\section{CONCLUSION}

In this paper, the nonlinear characteristics of the periodic forced system with soft impacts were analyzed with special attention on stability of periodic motion, grazing bifurcation, period-doubling bifurcation, Hopf bifurcation and chaotic motion, etc. The piecewise properties and routes to chaos have been shown as follows.

(1) The 1-1-1 motion, in most cases, underwent grazing bifurcation or Hopf bifurcation to chaos with a change in the system parameters.

(2) Grazing bifurcation led to singularity and asymmetric periodic motion, such as 4-3-4 motion, 6-3-4 motion 3-1-3 motion,.

(3) The clearances of the system are the main reasons for influencing frequency range of periodic motions and chaos.

(4) The steady 1-1-1 period orbits existed within a wideband frequency range and the value of velocity achieved the desired results when appropriate system parameters were chosen.

\section{CONFLICT OF INTEREST}

The authors confirm that this article content has no conflict of interest. (a) $b=0.0$

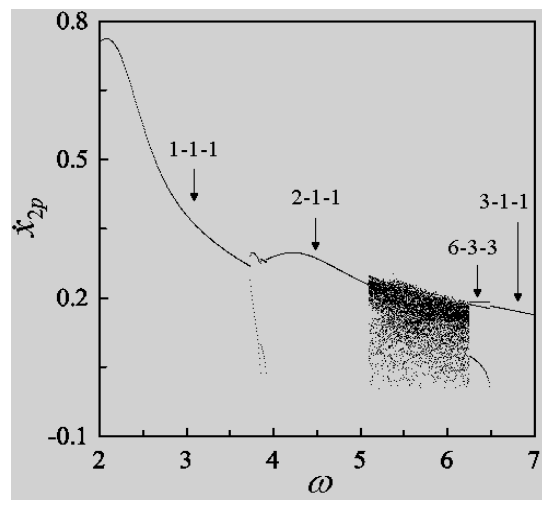

(b) $b=0.02$

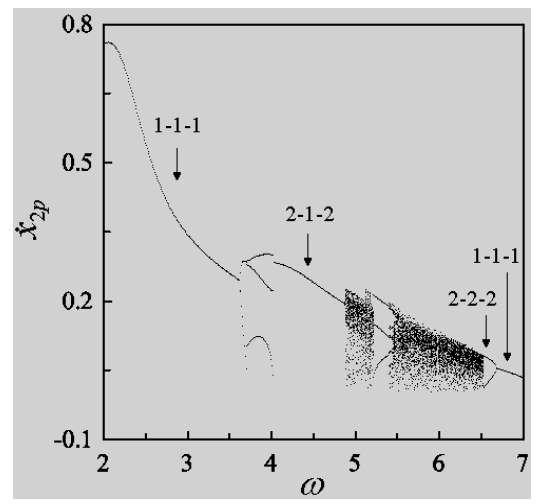

(c) $b=0.05$

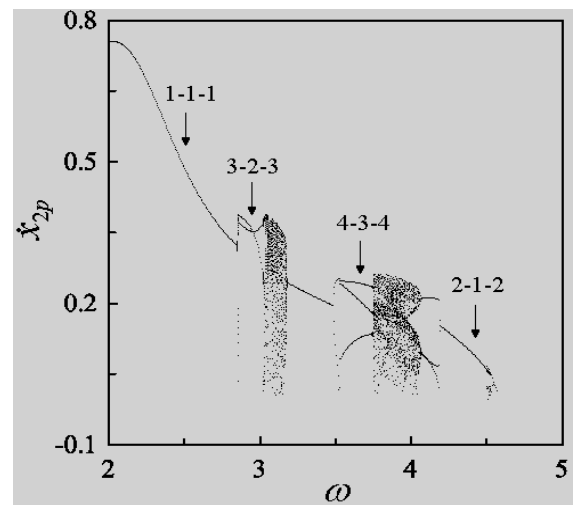

(d) $b=0.1$

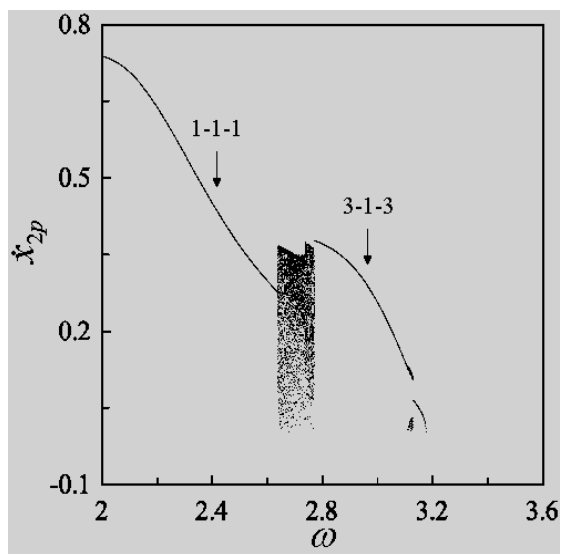

Fig. (6). Bifurcation diagrams associated with the clearances. 


\section{ACKNOWLEDGEMENTS}

The authors gratefully acknowledge the support by Youth scientific funds of the Lanzhou Jiaotong University (2013024), Natural Science Foundation of Gansu Province (145RJZA056) and Pre-research funds of Jinchuan Group CO., LTD. (420032).

\section{REFERENCES}

[1] S. W. Shaw, and P. J. Holmes, "A periodically forced piecewise linear oscillator", Journal of Sound and Vibration, vol. 90, pp. 129-155, 1983.

[2] A. B. Nordmark, "Non-periodic motion caused by grazing incidence in an impact oscillator", Journal of Sound and Vibration, vol. 145, pp. 279-297, 1991.

[3] S. L. T. De Souza, and M. Wiercigroch, "Suppressing grazing chaos in impacting system by structural nonlinearity", Chaos, Solitons and Fractals, vol. 38, pp. 864-869, 2008.

[4] X. H. Long, G. Lin and B. Balachandran, "Grazing bifurcations in an elastic structure excited by harmonic impactor motions", Physica D, vol. 237, pp. 1129-1138, 2008.

[5] A. C. J. Luo, and B. C. Gegg, "Grazing phenomena in a periodically forced, friction-induced, linear oscillator", Communications in Nonlinear Science and Numerical Simulation, vol. 11, pp. 777-802, 2006.
[6] M. Wiercigroch, and V.T.W. Sin, "Experimental study of a symmetrical piecewise base-excited oscillator", ASME Journal of Applied Mechanics, vol. 65, pp. 657-663, 1998.

[7] A. C. J. Luo, and D. O'Connor, "Periodic motions and chaos with impacting chatter and stick in a gear transmission system", International Journal of Bifurcation and Chaos, vol. 19, pp. 1975-1994, 2009

[8] A. C. J. Luo, and D. O'Connor, "Mechanism of impacting chatter with stick in a gear transmission system", International Journal of Bifurcation and Chaos, vol. 19, pp. 2093-2105, 2009.

[9] G.W. Luo, J.N. Yu, H.M. Yao, and J.H. Xie, "Periodic-impact motions and bifurcations of the vibratory system with a clearance", Chinese Journal of Mechanical Eengineering, vol. 42, pp. 88-94, 2006.

[10] E.V. Karpenko, M. Wiercigroch, E.E. Pavlovskaia, and R.D Neilson, "Experimental verification of Jeffcott rotor model with preloaded snubber ring", Journal of Sound and Vibration, vol. 298, pp. 907-917, 2006

[11] A. Al-shyyab, and A. Kahraman, "Non-linear dynamic analysis of a multi-mesh gear train using multi-term harmonic balance method: sub-harmonic motions", Journal of Sound and Vibration, vol. 279, pp. 417-451, 2005.

[12] G.W. Luo, and H.M. Yao, "Dynamics of a small vibro-impact pile driver", Nonlinear Analysis: Real World Applications, vol. 9, pp. 1361-1377, 2008.

(C) Zhu and Gao; Licensee Bentham Open.

This is an open access article licensed under the terms of the Creative Commons Attribution Non-Commercial License (http://creativecommons.org/licenses/by-nc/4.0/) which permits unrestricted, non-commercial use, distribution and reproduction in any medium, provided the work is properly cited. 